\title{
A new technique for adjusting distraction moments in multitasking non-field usability tests
}

\author{
Gregor McGlaun, Frank Althoff, Björn Schuller, and Manfred Lang \\ Institute for Human-Machine-Communication, \\ Technical University of Munich, \\ 80290 Munich, Germany \\ \{mcglaun, althoff, schuller, lang\}@ei.tum.de
}

\begin{abstract}
Evaluating errors that result from user interactions with incar applications, it has to be considered that the user is permanently involved with driving the car. Reproducing this driving workload in non-field usability tests, it showed that the driving simulation demanded each test subject in a different way because of individual precognitions and properties. To ensure an identical driving workload for each test subject, it becomes necessary to individually adapt the degree of difficulty (DOD) of the driving task. We present a new technique in which, concerning the driving performance, each test participant is pre-classified in a baseline investigation before the main trial. In this context, a special measurement for objectively validating the driving performance of the subjects is being introduced.
\end{abstract}

\section{Keywords}

Usability Engineering, distraction task, driving task, operation task, workload, human error, pre-classification

\section{INTRODUCTION}

With regard to the evaluation of errors in the humanmachine interaction in the automotive environment, a substantial problem is the permanent superposition of several user acts. Implicated by the domain, the user has to execute all operations concerning the in-car applications simultaneously with driving the car. To meet these facts in non-field usability studies, the participants have to perform in a 3D-driving task in addition to the operation task to be evaluated. Yet, it often showed that a set of participants had nearly no difficulty with the driving task, whereas others were demanded so much that they were hardly able to operate the in-car application. Subjects of the latter group showed the tendency to no longer take the driving task seriously, and solely concentrated on the operation of the in-car application. To avoid these unrealistic scenarios, the design of this kind of trials requires a rather sensitive taxonomy of the specific influence factors. The participant with her or his personal properties and individual precognitions, the DOD of the driving task simulation, and

Copyright is held by the author/owner(s)

CHI 2002, April 20-25, 2002, Minneapolis, Minnesota, USA.

ACM 1-58113-454-1/02/0004.

This is the author's version of the work. It is posted here for

your personal use. Not for redistribution. the scope of the operation task form a barycentrical triangle that has to be kept constant over the whole progression of the trial. In terms of the operation task, the driving thread represents a distraction moment. To reproduce the test scenarios in equal terms, it is necessary to ensure a distraction moment that is not too powerful and, above all, identical for each test subject.

\section{METHODOLOGY}

Implementation in a Test Concept

Before the main test, each test person participates in a socalled baseline investigation in which the subject is preclassified with regard to his driving performance. Within the baseline investigation, which runs over a period of 180 s, the participant is not yet confronted with any kind of operation task. The subject has to follow a predefined course of a road that is unknown to her or him. For reproducibility reasons, in this ascertainment, the participant cannot manipulate the velocity, but by a predefined run chart, the speed of the car is varied automatically between five DODs. The degrees are characterized by the frequency and the narrowness of the curves and the speed. In general, the time slots in the sequence of the DODs are of different length, and the levels are not monotonously de- or increasing to eliminate anticipation effects by the test persons. The concrete driving task is to always keep the car on the right lane of the street. This means, the vertex of the triangle $T$ (representing the current position of the car) must be kept within the limits (distance $q$ ) given by the broken white line $A$ in the middle of the road and the right road side $B$ (c.f. 1).

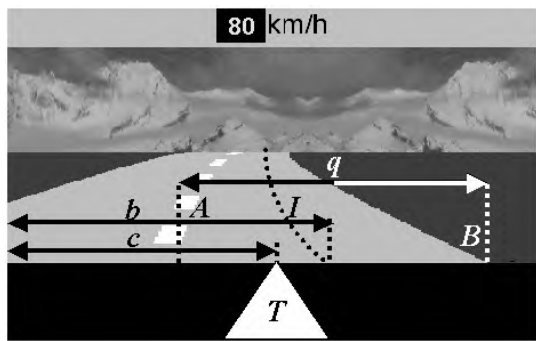

Figure 1: screenshot of the 3D-driving simulation

During the whole study, the absciss $c$ (c.f. 1) of the position of the vertex of $T$ in relation to the absciss $b$ of the ideal line $I$ (c.f. 1) and the correspondent timestamps $t$ are 
recorded in a log file (at a sample rate of $13 \mathrm{~Hz}$. Directly after the baseline investigation, the test subject is preclassified, and, based on the result, the DOD of the driving task in the main trial is determined. If a participant had a bad performance in the baseline investigation, the DOD will be respectively low in the main part. To objectively rate the driving performance of each test subject objectively, a special numerical validation measurement based on the driving data is introduced.

\section{Computation of the Error Score}

If the driver leaves the right lane of the road, i.e. in case the condition $|c-b|-0.5 q<0$ is violated, a so-called deviation event $D_{j}$ is generated. Then, $d=|c-b|-0.5 q$ represents the distance of the car from the boundary of the lane in the simulation. Let $j$ be a counting index for the deviation events, $i$ for the distance samples, $\mathrm{m}(j)=\left|D_{j}\right|$, and $d_{l, 1}$ denote the first recorded distance of the first deviation event $D_{l}$. Then, a deviation event $D_{j}$ can be characterized by a set of distance samples, as follows: $D_{j}=\left\{d_{1, j}, d_{2, j}, \ldots\right.$, $\left.d_{m(j), j}\right\}$. Each deviation event is validated with a numerical value $E_{j}$. This measurement is dependent on the individual performance factors:

i) the current distance $d_{i j}$ at the timestamp $t_{i j}$ and

ii) the DOD $s_{i j}$, at the timestamp $t_{i j}$.

The $j$-th contribution $E_{j}$ of one deviation event $D_{j}$ is computed as the linear approximation of the area between the edge of the lane (A, and $\mathrm{B}$, respectively, ) and the driven line. Assumed the car has exceeded the left border $A$, in figure 2, an exemplary visualization for the computation of $E_{j}$ is given (bird view, scene rotated by $-90^{\circ}$ ):

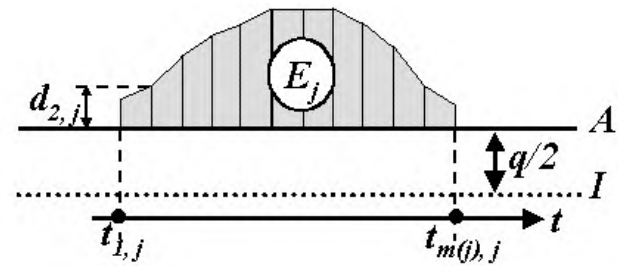

Figure 2: visualization of the computation of $E_{j}$

Thus, $E_{j}$ can be computed via the following formula:

$$
E_{j}=\mathfrak{R}_{i=2}^{m(j)} \frac{\left(t_{i, j}-t_{i-1, j}\right)\left(d_{i, j}+d_{i-1, j}\right)}{2} .
$$

Assumed $n$ deviation events, the total error score $E^{*}$ is calculated as the weighted sum of all $n$ score contributions. The weight of each single deviation is determined by $s_{1, j}$ which is the DOD having been effective at the time $t_{l, j}$ :

$$
E^{*}=\mathfrak{R}_{j=1}^{n} \frac{E_{j}}{s_{1, j}}=\mathfrak{R}_{j=1}^{n} \underset{i=2}{\boldsymbol{m}(j)} \frac{\left(t_{i, j}-t_{i-1, j}\right)\left(d_{i, j}+d_{i-1, j}\right)}{2 s_{1, j}} \text {. }
$$

With $E^{*}$ being a monotonously increasing function of $n$, it is a direct measurement for the user performance in the driving task, as the contributions of the deviation areas are all non-negative).

\section{Classification Scheme}

For the classification of the test subjects, the score $E^{*}$ is compared with values of a look-up table (LUT). The LUT consists of several score intervals. To each interval, a correspondent DOD of the driving task to be used in the main task is assigned. The single score intervals of the LUT depend on peripheral constants which are

i) the determination of the course of the street,

ii) the determination of the particular speed levels,

iii) the width $r$ of the car, and

iv) the width $q$ of the right lane

Based on the results In a preliminary study, which will be described elsewhere, the LUT (see table 1) was determined on the following conditions: resolution $1024 \times 768$ pixels, $r=1$ pixel (car represented by vertex of a triangle), $q=380$ pixels, DOD $1=$ easy,..., DOD $5=$ hard:

\begin{tabular}{|l|l|l|l|l|l|}
\hline $\begin{array}{l}\text { Error score (in } \\
\text { ms*pixels) }\end{array}$ & $0-3000$ & $\begin{array}{l}3001- \\
6000\end{array}$ & $\begin{array}{l}5001- \\
7000\end{array}$ & $\begin{array}{l}7001- \\
9000\end{array}$ & $9001-$ \\
\hline Level & 5 & 4 & 3 & 2 & 1 \\
\hline
\end{tabular}

Table 1: LUT for the determination of the DOD

\section{RESULTS AND CONCLUSIONS}

The presented technique proved its efficiency in several tests series (see also [1]). In the latest one, 15 subjects (from the age of 20 to 65 , average age 32 ) participated. The frequency of the particular DODs is given in brackets after the DOD itself 5(5), 4(3), 3(2), 2(4), 1(1). The average DOD on all subjects determined was 3.5 . The large mean variation showed that a pre-classification is necessary and not negligible in this kind of investigations. Exclusively regarding the results of the subjects in the eldest group (age over 50), which consisted of 5 participants, we got the distribution as follows: 4(1), 2(3), and 1(1), with gives an average level of 2.2. On the other hand, the youngest group (5 subjects) had 5(3) and 4(2), which is 4.6 on average. This shows that especially elderly people are demanded more by the driving task. In further works, the logged driving data will be explicitly evaluated with regard to the driven curve of the participant in relation to the ideal line. Thus, we hope to be able to make special statements about the influence of the operation task on the driving performance of the subjects.

\section{ACKNOWLEDGEMENTS}

The work presented in this paper has been supported by the FERMUS-project, which is a cooperation between the BMW Group, SiemensVDO AG, DaimlerChrysler AG, and the Institute of Human-Machine-Communication at the Technical University of Munich. FERMUS stands for error robust multimodal speech dialogues.

\section{REFERENCES}

1. G. McGlaun et al.: "A generic operation concept for an ergonomic speech MMI under fixed constraints in the automotive environment," $9^{\text {th }}$ Int. Conference on HCI 2001, New Orleans 